\title{
Neoplasia vesical tras nefroureterectomia por tumores de urotelio superior. ¿La cirugía del uréter distal influye en la evolución?
}

\author{
Blanco Díez A, Armas Molina J, Limiñana Cañal JM**, Artíles Hernández JL, Martín Betancor D, \\ Chesa Ponce N.
}

\author{
Servicio de Urología. *Unidad de Investigación. Hospital Universitario Insular. Las Palmas de Gran Canaria.
}

\author{
Actas Urol Esp. 2007;31(1):23-28
}

\section{RESUMEN}

NEOPLASIA VESICAL TRAS NEFROURETERECTOMÍA POR TUMORES DE UROTELIO SUPERIOR. ¿LA CIRUGÍA DEL URÉTER DISTAL INFLUYE EN LA EVOLUCIÓN?

Objetivo. Comparar la evolución de los pacientes con tumor de urotelio superior (TUS) en los cuales realizamos, durante la nefroureterectomía, abordaje clásico abierto de desinserción del uréter distal con aquellos en los que realizamos abordaje endoscópico. Estudiamos el indice de recurrencias vesicales en cada grupo y los factores que pueden influir en la evolución.

Material y métodos. Se realiza una revisión retrospectiva de las nefroureterectomías realizadas en nuestro Servicio en los últimos 20 años. Se dividen en dos grupos. Grupo A (n: 29): doble incisión (lumbar y pélvica), Grupo B (n: 24): abordaje endoscópico del uréter distal e incisión lumbar clásica.

Se realiza en primer lugar un estudio descriptivo en el que se expone: sexo y edad del paciente, antecedentes de resección vesical previa por tumor (RTU-V), lado del TUS, y localización (cálices, pelvis o uréter). También revisamos estadio patológico y grado tumoral.

En segundo lugar se realiza una revisión de las recidivas vesicales en cada grupo.

Para establecer posibles diferencias utilizamos el Test exacto de Fisher.

Resultados. Observamos diferencias estadísticamente significativas en términos de recurrencias vesicales a favor del grupo B ( $<<0.036)$, lo que significa que existen menos recidivas vesicales en nuestra serie en el grupo de cirugía endoscópica. Sin embargo debido a la inhomogeneidad de los grupos en estadio, grado y localización tumoral, esta diferencia parece más bien atribuible a estas características, más que a la vía de abordaje como factor independiente.

En lo referente a la relación entre la recidiva vesical y antecedentes de RTU-V no hemos encontrado diferencias entre los grupos pero si existe diferencia en la serie global. El escaso número de recurrencias (13) no nos permite establecer una conclusión clara en este problema, aunque parece que el antecedente de neoplasia previa de vejiga influye en la posibilidad de recurrencia vesical.

Conclusiones. En nuestro resultado el abordaje endoscópico en la nefroureterectomía por TUS parece influir en la posterior aparición de menos recidivas en vejiga, sin embargo la muestra es corta y los grupos no son homogéneos porque el abordaje del uréter no se ha aleatorizado por razones éticas obvias. Si parece existir relación entre la existencia de antecedentes de RTU-V y la aparición de recidivas posteriores, como mostramos en la serie global. En cualquier caso son necesarios estudios prospectivos randomizados multicéntricos para conocer si el abordaje del uréter distal influye en la posterior aparición de recidivas vesicales.

Palabras clave: Cáncer de vejiga. Nefroureterectomía. Cirugia. Desinserción ureteral.

\section{ABSTRACT}

\section{BLADDER NEOPLASM AFTER NEPHROURETERECTOMY FOR UPPER URINARY TRACT TUMOR. DOES DISTAL URETER SURGERY} INFLUX ON THE EVOLUTION?

Objective. To compare evolution in patients with urothelial upper tract tumor (UUTT) in whom we performed the classic open approach to the distal ureter with those in whom whe performed the endoscopic approach. We study the bladder recurrences in each group and the factors which may influx on the evolution.

Material and methods. Retrospective review of nephroureterectomies in our department in the last 20 years. Group A (n:24): double incision (lumbar and pelvic incision), Group B (n:29): endoscopical approach of the distal ureter and classic lumbar incision.

A descriptive study is first performed in which we review: sex, and patients age, background transuretral resection of bladder tumor (TURBT), UUTT side, and UUTT location (calices, pelvis or urether). Pathological stage and tumor grade are also exposed.

Secondly, a review of the bladder recurrences in each group is performed

In order to find differences between de groups we used the Fisher's Exact test.

Results. We observed that in terms of bladder recurrence there is a statistically significant difference between the two groups favoring Group B ( $<$ 0.036), which means that there are less bladder recidives when perform endoscopic approach of distal ureter. Noneless due to the groups inhomogeneity in tumor stage, grade, and location, this differences seem to be associated to these sigues, more than to the approach to the ureter as an independant variable.

In that concerning to the eventual relationship between bladder recidive and background of previous TURBT, we have not found any differences between the two groups but there are statystically significant difference in the global series. The small number of recurrences (13) does not allow us to establish a well based conclusion on this issue, but it seems that the background of previous TURBT is an important factor that may influx in posterior bladder recurrences.

Conclusions. In our results, endoscopic approach of distal ureter in nephroureterectomy for UUTT does seems to positively influx in the posterior chance of bladder recurrences. But the studied sample is small and the two groups have not been aleatorized because of ethical reasons. May the low number of cases affect the test's statistical potency. It seems that previous TURBT may conditionate these recurrences, as we show in global serie. It should be necessary a prospective and statistically analised multicenter trial to understand if distal ureter approach influx on the evolution of these tumors.

Keywords: Bladder cancer. Nephroureterectomy. Surgery. Ureteral desinsertion. 
$\mathrm{L}$ a nefroureterectomía es el tratamiento estándar para el manejo de los TUS. Tradicionalmente la técnica quirúrgica incluía dos incisiones: una lumbar para la nefrectomía y otra iliopélvica (Gibson), para la desinserción del uréter distal. En 1952, Mc Donald ${ }^{1}$, publica en el Journal of Urology, una nueva técnica de desinserción endoscópica, que permite extraer el espécimen por vía lumbar, y extirpar el uréter en su totalidad evitando la segunda incisión.

Hay sin embargo cierta controversia sobre la posibilidad de implante tumoral alrededor del orificio ureteral y retroperitoneo, cuando se utiliza esta técnica, habiéndose descrito la posibilidad de recidivas vesicales.

Nosotros exponemos nuestra experiencia en nefroureterectomías y comparamos evolución en pacientes en los que se realizó el abordaje clásico con aquellos en los cuales lo hicimos vía endoscópica. Estudiamos las recurrencias vesicales en cada grupo y los factores que pueden influir en la evolución. No tuvimos recurrencias en área de desinserción ni en retroperitoneo en nuestra serie.

Por último realizamos una revisión bibliográfica para intentar arrojar luz sobre este tema.

\section{MATERIAL Y MÉTODOS}

Hemos realizado una revisión retrospectiva de las nefroureterectomías por TUS entre 1982 y 2002. Estudiamos aquellos con seguimiento posterior de al menos 24 meses. Se dividen los pacientes en dos grupos: Grupo A: doble incisión (lumbar y pélvica), Grupo B. desinserción endoscópica del uréter. En el primer caso la cirugía fue siempre la misma. En un primer tiempo nefrectomía por vía lumbar y posteriormente incisión ilioinguinal de Gibson extraperitoneal para disecar uréter hasta pared vesical. En este momento se tira del uréter haciendo "tienda de campaña "y se reseca por completo con pastilla vesical. Se sutura por último el defecto vesical. En cuanto al manejo endoscópico, fue siempre el mismo: circuncisión perimeática con Asa de Collins hasta grasa perivesical, previa coagulación del la luz ureteral. Se continúa la intervención tras colocar una sonda vesical, con una lumbotomía, y nefrectomía disecando el uréter hasta liberarlo y extrayendo el espécimen completo con pastilla vesical. La realización de uno $u$ otro procedimiento obedece a criterios cirujano dependiente, y localización del tumor dependiente, realizándose desinserción endoscópica en casi todos los tumores de cáliz y pelvis y sólo en 2 de los localizados en uréter distal.

Se realiza un estudio descriptivo previo en el que se revisa: sexo y edad de los pacientes, antecedentes de RTU-V, lado del TUS y localización (cálices, pelvis o uréter). Asimismo se revisa estadio y grado tumoral.

Posteriormente hacemos una revisión de las recurrencias vesicales en cada grupo. Consideramos especialmente el antecedente de RTU-V, las muertes cáncer específicas y las pérdidas de seguimiento.

Por último se realiza un análisis estadístico para determinar diferencias entre los dos grupos en términos de recurrencias vesicales, así como recurrencias relacionadas con antecedentes de RTU-V. Utilizamos el programa SPSS 13.0 con el Test exacto de Fisher (dado que las muestras son pequeñas) para analizar los datos.

\section{RESULTADOS}

Estudiamos 53 pacientes sometidos a nefroureterectomía. El Grupo A: 29 pacientes, Grupo B: 24 pacientes. De estos, 10 habían sido tratados antes con RTU-V por neoplasia vesical, 6 en el primer Grupo y 4 en el segundo. El seguimiento medio del Grupo A fue de 62 meses (rango: 24200), y del B 59 meses (rango: 28-196). Del total, $47(88,6 \%)$ son hombres, y 6 mujeres $(11,4 \%)$. La media de edad es de 62,2 años (rango: 47-78). En el lado derecho encontramos 28 tumores $(52,8 \%)$, y $25(47,2 \%)$ en el izquierdo. En todos los casos del Grupo B, se realizó la técnica de desinserción endoscópica descrita. Los datos descriptivos de situación del TUS, estadio tumoral y grado tumoral se muestran en la Tabla 1.

Como podemos observar, del total de los tumores ureterales, sólo dos casos (13\%) se sometieron a liberación endoscópica, hecho destacable para la comprensión de los resultados, debido a la ausencia de homogeneidad en la muestra en este hecho. Tampoco existe homogeneidad en lo referente al estadio tumoral habiendo el doble de tumores infiltrantes en el grupo A que en el B. 
Tabla 1

Datos descriptivos de los TUS

\begin{tabular}{lccc}
\hline & Total & Grupo A & Grupo B \\
\hline Cálices y pelvis & 25 & 7 & 18 \\
Pelvis y uréter & 13 & 9 & 4 \\
Uréter & 15 & 13 & 2 \\
pT1 & 31 & 15 & 16 \\
pT2: & 12 & 9 & 3 \\
pT3: & 10 & 5 & 5 \\
GI & 2 & 2 & 0 \\
GII & 35 & 17 & 18 \\
GIII & 16 & 10 & 6 \\
\hline
\end{tabular}

En lo que se refiere a las recurrencias vesicales: 13 pacientes (24\% del total). De estos, 9 en el Grupo A, (31\% del total del grupo) y 4 en el Grupo B (16,6\% del total del grupo). En lo relativo al antecedente de RTU-V vemos que de 9 recurrencias en grupo A 6 habían sido anteriormente resecadas por tumor vesical (66,6\%). En Grupo $B, 3$ que recurrieron (75\%) tenía dicho antecedente.

Del total, 4 pacientes fueron perdidos para seguimiento, aunque todos ellos fueron seguido durante 24, 28, 38, 39 meses respectivamente los tres primeros en Grupo A y 1 en B. Hubo 8 muertes tumor-relacionadas, 6 en Grupo A, y 2 en B, no relacionadas con el procedimiento, sino posiblemente con el estadio (había 5 T3) y con el grado (los 8 eran GIII).

Una vez realizado el análisis estadístico observamos que existía una diferencia estadísticamente significativa entre los dos grupos $(p<0,036)$ a favor del B lo cual significaría que existen menos recidivas vesicales cuando se realiza abordaje endoscópico del uréter distal. Sin embargo debido a las diferencias entre los dos grupos en término de estadio, grado y localización tumoral cabe afirmar que la técnica quirúrgica no parece una variable independiente en la evolución de la enfermedad, sino que es una más en el conjunto de variables.

En lo referente a la relación entre la recurrencia vesical y el antecedente de RTU-V, no se encuentran diferencias entre los grupos $(\mathrm{p}<0,670)$, pero si existe diferencia estadísticamente signifi- cativa en la serie global. De 13 pacientes que recurrieron, 9 (69,2\%) tenían antecedentes de neoplasias vesicales previamente resecadas, lo que nos permite pensar en la posible relación entre el antecedente de RTU-V previa y la posterior recidiva. El escaso número de recurrencias no nos permite establecer una conclusión clara en este tema.

\section{DISCUSIÓN}

Los TUS constituyen menos del 5\% de los tumores uroteliales, y son tumores con un amplio espectro de patrones de conducta. En el $20-30 \%$ de los casos existe una historia de neoplasia vesical y en el $2.5 \%$ un tumor sincrónico bilateral. Son más frecuentes en aquellos pacientes tratados previamente de tumores vesicales. Algunas de las razones serían las RTU-V repetidas y el reflujo vésico-ureteral ${ }^{2}$.

Estas neoplasias se comportan grado por grado y estadio, por estadio de la misma manera que las vesicales.

Existen evidencias de que los TUS se implantan de arriba hacia abajo. Existen varios trabajos que exponen la alta incidencia de recurrencias en el muñón ureteral después de una nefroureterectomía incompleta, así como la recurrencia en orificio ureteral. También ha sido comentado que los TUS rara vez se desarrollan por encima del nivel de resección de un tumor ureteral ${ }^{2-4}$.

La nefroureterectomía es la técnica "gold standard" cuando un TUS no cumple criterios para cirugía de conservación. El espécimen debe incluir el uréter y la pastilla vesical periorificial (Kimball y Ferris $^{5}$ ). Esta técnica se realiza para tratar la lesión local de una forma más efectiva debido a la posibilidad de multicentricidad de estos tumores como lo describió Carron en $1982^{6}$. Incluso aunque el grado y estadio tumoral son los factores más importantes en la determinación de la supervivencia a largo plazo, otros pueden influir, como la multiplicidad y el abordaje quirúrgico ${ }^{2}$.

La dificultad en analizar la técnica endoscópica es debida al diseño retrospectivo de las series publicadas así como a la imposibilidad de randomización, la baja frecuencia de los TUS, y las selectivas indicaciones para el manejo endoscópico de los tumores de uréter distal. 
Las recidivas tras nefroureterectomía no son infrecuentes (23-30\%). Sin embargo su incidencia aumenta cuando existe una historia previa de tumor vesical, relacionándose de forma directa con el grado tumoral del tumor primario. Hasta el $80 \%$ de las recurrencias vesicales aparecen en los primeros 2-3 años tras el tratamiento del primario. En el $90 \%$ de los casos tiene el mismo grado y concuerda en estadio en el $72 \%{ }^{9}$. No existen estudios serios sobre la cirugía abierta que indiquen la zona de mayor recurrencia, que parece más frecuente en trígono ipsilateral. No hay diferencia en términos de recurrencias vesicales entre las series clásicas de cirugía abierta y las más recientes de cirugía endoscópica ${ }^{2}$.

En esta revisión encontramos 4 estudios que comparan ambas técnicas ${ }^{10-13}$. Angulo et al. refieren tiempo operatorio y estancia media significativamente más bajos cuando se hace desinserción endoscópica. El sangrado también es menor pero no estadísticamente significativo. Zubac $^{10}$ confirma el beneficio de dicho abordaje en relación a estancia hospitalaria y supervivencia. Todas estas series amparan la eficacia y radicalidad de esta técnica en pacientes bien seleccionados. Sin embargo el análisis de Saica ${ }^{11}$ sobre 60 pacientes de los cuales 28 fueron sometidos a abordaje endoscópico del uréter distal y 32 a cirugía abierta concluye que aunque el tiempo operatorio y la pérdida de sangre son menores en el primer grupo, el índice de recurrencias fue más alto en el primer grupo con diferencias estadísticamente significativas.

Obviamente esta técnica tiene sus indicaciones y contraindicaciones, siendo fundamental la correcta selección de $\operatorname{casos}^{2-4,12,13,19}$. Estas son las contraindicaciones:

- Tumor en uréter iliopélvico

- Cirugía pélvica previa

- Radioterapia pélvica

- Aneurisma aórtico distal o iliaco

- Fibrosis retroperitoneal

- Tumor vesical sincrónico

La existencia de tumor en uréter distal, incrementa la probabilidad de recurrencias en área resecada, así como de implante retroperitoneal. Cuando existe antecedente de fibrosis retroperitoneal, aneurisma, o cirugía pélvica, el abordaje endoscópico debe ser evitado debido al riesgo de perforación peritoneal, que causaría implantes celulares en dicha cavidad. Incluso aunque poco frecuente puede darse la perforación de un aneurisma. La lesión peritoneal es bastante infrecuente dado que la resección del orificio ureteral se realiza en el área trigonal, pero las alteraciones anatómicas asociadas a las condiciones descritas pueden llevar a la lesión peritoneal. Generalmente debemos considerar que cualquier situación que altere la anatomía o funcionalidad de los tejidos pélvicos será una contraindicación del abordaje endoscópico ${ }^{3,4,5,7,20}$.

En lo que si existe acuerdo es en mostrar la vía endoscópica como la más rápida, con menos complicaciones, y más corta hospitalización. Pero hay que tener en cuenta un detalle: la importancia de extraer el uréter completo con el espécimen. Cuando se hace la desinserción, si esta no es completa, o la técnica no es correcta el uréter se romperá al tirar lo que constituye la causa más frecuente de la "reconversión"ha abierto del abordaje endoscópico ${ }^{14}$. Es también importante la coagulación del meato durante la desinserción, lo cual nos puede ayudar a reconocer el extremo ureteral durante la maniobra de "stripping", que nos asegura haberlo extraído en totalidad. Asimismo podría evitar implantes retroperitoneales en el área afectada ${ }^{2,3,12,13,17 . ~}$

En la revisión realizada leemos algún trabajo en el que se refiere la posibilidad de implante tumoral en el área resecada, intra o extraperitoneal. Sin embargo podemos preguntarnos si el abordaje abierto hubiera cambiado la evolución en estos casos concretos ${ }^{4,8,15,16}$.

Además de las series españolas mostradas $^{3,12,13}$ hay otras ${ }^{17-20}$. Todas reflejan datos semejantes, en lo relativo a menor tiempo quirúrgico, y estancia hospitalaria, así como seguridad oncológica. Una de ellas presenta un alto índice de recurrencias (50\%), pero no expone el posible antecedente de RTU-V en estos pacientes ${ }^{19}$. Mención especial nos merece el trabajo de Rodríguez Villamil ${ }^{18}$, por el tamaño muestral y el análisis estadístico. Muestra 145 pacientes sometidos a nefroureterectomía 60\% con doble incisión y $40 \%$ con desinserción endoscópica. No hubo diferencias estadísticamente significativas en número de recurrencias ni lugar de las mismas. Su conclusión es que el índice de recurrencias es independiente de la vía de abordaje. 
En este momento en el que la cirugía laparoscópica se impone como el presente de la cirugía urológica, nos podemos hacer esta pregunta. ¿Tiene todavía su papel la desinserción endovesical del uréter?. En 1993, Rassweiler ${ }^{21}$ expone este dilema, mostrando que la nefroureterectomía laparoscópica, puede ser asociada a la circuncisión meatal transuretral. Tras seguimiento de 4 años no se observan recurrencias. La gran ventaja de la laparoscopia es que podemos conseguir una buena visión de todo el uréter hasta el trayecto intramural, consiguiendo por tanto una buena hemostasia. Mc Neill en 2002, también defiende la nefroureterectomía laparoscópica, realizando la desinserción endoscópica por una incisión de Pfannestield utilizada para extraer la pieza ${ }^{22}$. El problema del uréter distal es analizado en una revisión del M.D. Anderson en Houston, $\mathrm{TX}^{23}$. Admiten que aunque no existe evidencia de que un abordaje sea mejor que otro el procedimiento abierto parece más fácil y oncológicamente más eficaz. Por tanto los procedimientos endoscópicos deben ser utilizados con cuidado debido a la posibilidad de diseminación tumoral. La experiencia de la Washington University es importante dada la amplia casuística expuesta, y también apoya la vía endoscópica dando un papel primordial a la vía laparoscópica ${ }^{24}$.

Como conclusión pensamos que una selección correcta de los pacientes, una técnica bien realizada $^{3,4,7}$, con coagulación previa del meato, y un manejo cuidadoso del espécimen durante la nefrectomía, hace esta vía segura y oncológicamente radical ${ }^{12,25,26}$. Es muy importante para nosotros recordar que los condicionantes más claros para la recurrencia o el implante tumoral son: características del tumor (grado y estadio), y la histología de la neoplasia vesical previa. En este caso la recurrencia tendrá más relación con la neoplasia vesical que con el TUS.

\section{CONCLUSIONES}

En nuestra serie el grupo en el que se realiza el abordaje endoscópico tiene menos recidivas vesicales, con una $\mathrm{p}<0,036$. Sin embargo las diferencias entre los grupos en estadio, grado, y localización tumoral, hacen las dos muestras no homogéneas, y no podemos establecer la vía de abordaje del uréter como variable independiente en la evolución de estos tumores. Si parece existir relación entre la existencia de antecedentes de RTU-V y la aparición de recidivas posteriores, como mostramos en la serie global. En cualquier caso son necesarios estudios prospectivos randomizados multicéntricos para conocer si el abordaje del uréter distal influye en la posterior aparición de recidivas vesicales.

\section{REFERENCIAS}

1. Mc Donald HP, Upchurch WE, Sturdevant CE. Nephroureterectomy: a new technique. J Urol. 1952;67(6):804-809.

2. Laguna MP, De la Rosette JJ. The endoscopic approach to the distal urether in nephroureterectomy for upper urinary tract tumour. J Urol. 2001;166(6):2017-2022.

3. Palou J, Caparros A, Orsola B, Xabier B,Vicente J. Transurethral resection of the intramural ureter as the first stage of nephroureterectomy J Urol. 1995;154(1):43-44.

4. Fernandez Gomez JM, Escaf Barmadah S, Perez Garcia J, Rabade Rey CJ, Rodriguez Martinez JJ. Riesgo de implante tumoral tras nefroureterectomía con resección endoscópica de meato ureteral. Arch Esp Urol. 1998;51(8):829831 .

5. Kimball FN, Ferris HW. Papilomatous tumour of the renal pelvis associated with similar tumours of the ureter and bladder: review of literature and report of two cases. J Urol. 1934;31(4):257-304.

6. Mc Carron P, Chasko SB, Gray GF. Systematic mapping of nephroureterectomy specimens removed for urothelial cancer: pathological findings and clinical correlations $\mathrm{J}$ Urol. 1982;128(2):243-246.

7. Abercrombie GF, Eardley I, Payne SR, Walmsley BH, Vinnicombe J. Modified nephroureterectomy. Long term follow-up with particular reference to subsequent bladder tumours. Br J Urol. 1988;61(3):198-200.

8. Hetherington JW, Ewing R, Philip NH. Modified nephroureterectomy: a risk of tumour implantation”. Br J Urol. 1986; 58(4):368-370.

9. Krogh J, Kvist E, Rye B. Transitional cell carcinoma of the upper urinary tract: prognosis variables and postoperative recurrences. Br J Urol. 1991;67(1):32-36.

10. Zubac DP, Kihl B. One or two incisions for nephroureterectomy in transitional cell renal pelvis tumours. Scand J Urol Nephrol. 1997;31(5):431-433.

11. Saika T, Nishiguch J, Tsushima T, Nasu Y, Nagai A, Miyaji $\mathrm{Y}$, et al. Comparative study of ureteral stripping versus open ureterectomy for nephroureterectomy in patients with transitional carcinoma of renal pelvis. Urology. 2004; 63(5):848-852.

12. Salvador Bayarri J, Rodriguez Villamil L, Imperatore V, Palou Redorta J, Villavicencio Mavrich H, Vicente Rodriguez J. Bladder neoplasms after nephroureterectomy: does the surgery of the lower ureter, transurethral resection or open surgery, influence the evolution?. Eur Urol. 2002; 41(1):30-33.

13. Angulo JC, Hontoria J, Sanchez Chapado M. One incision nephroureterectomy endoscopically assisted by ureteral stripping. Urology, 1998;52(2):203-207.

14. Giovansili B, Peyromaure M, Saighi D, Dayma T, Zerbib M, Debre D. Stripping technique for endoscopic management of distal ureter during nephroureterectomy: experience of 32 procedures. Urology. 2004;64(3):448-452. 
15. Jones DR, Moisey CU. A cautionary tale of the modified "pluck" nephroureterectomy. Br J Urol. 1993;71(4):486.

16. Arango O, Bielsa O, Carles J, Gelabert-Mas A et al. Massive tumor implantation in the endoscopic resected area in modified nephroureterectomy. J Urol. 1997;157(5):1839.

17. Prats Lopez J, Prera Villaseca A, Abad Gairin C, Lopez Marti E. Nefroureterectomía simplificada. Nuestra experiencia. Comunicación al LVII Congreso Nacional de Urologia. Madrid 1992.

18. Rodriguez Villamil L, Salvador J, Imperatore V, Prados M, Millán F, Vicente J. Tumor vesical tras nefroureterectomía en relación a la técnica de desinserción ureteral. Comunicacion al LXIII Congreso Nacional de Urología. Madrid 2000.

19. Pascual Regueiro D, Trivez Boned MA, Garcia Garcia MA, Garcia de Jalon Martinez A, Blas Marin M, Rioja Sanz LA. Desinserción ureteral endoscópica en nefroureterectomía radical: presentación de nuestra serie. Actas Urol Esp. 2002;26(2):85-90.

20. Polo Peris AC, Gonzalvo Perez V, Navarro Anton JA, Botella Almodovar R, Canto Faubel E, Gasso Matoses M et al. Desinserción ureteral endoscópica previa a nefroureterectomía. Análisis preliminar. Actas Esp Urol. 1998;22(7): 595-598.

21. Rassweiler JJ, Henkel TO, Potempa DM, Copcoat M, Alken P. The technique of transperitoneal laparoscopic nephrectomy, adrenalectomy and nephroureterectomy. Eur Urol. 1993;23(4):425-430
22. Mc Neill SA, Tolley DA. Laparoscopic nephroureterectomy for upper urinary tract transitional cell carcinoma. Arch Esp Urol. 2002;55(6):595-601.

23. Steimberg JR, Matin SF. Laparoscopic radical nephroureterectomy: dilemma of the distal ureter. Curr Opin Urol. 2002; 14(2):61-65.

24. Shalhav AL, Dunn MD, Portis AJ, Elbahnasy AM, Mc Dougall EM, Clayman RV. Laparoscopic nephroureterectomy for upper tract transicional cell cancer: the Washington University experience. J Urol. 2000;163(4): 1100-1104.

25. Kural AR, Demirkesen O, Arar O, Onder AU, Yalcin V, Solok V. Modified "pluck" nephroureterectomy for upper urinary tract disorders: combined endourologic and open approach. J Endourol. 1997;11(2):131-134.

26. Shental J, Rozenman J, Chaimowitch G, Almog D. Nephroureterectomy through a single lumbar incision combined with endoscopic incision of a bladder cuff. Urol Int. 1999;62(3): 147-149.

Correspondencia autor: Dr. A. Blanco Díez. Servicio de Urología. Hospital Universitario Insular. Plza. del Dr. Pasteur, s/n. 35016 Las Palmas de Gran Canaria. Tel.: 928444000.

E-mail autor: abdiez2003@yahoo.es

Información artículo: Original - Cáncer urotelio superior (Trabajo aceptado octubre 2006) 